\title{
Trends in the curative treatment of localized prostate cancer after the introduction of prostate-specific antigen: data from the Rotterdam Cancer Registry
}

\author{
S.J.J.C. SPAPEN, R.A.M. DAMHUIS and W.J. KIRKELS* \\ Comprehensive Cancer Centre, and *Department of Urology, Academic Hospital Dijkzigt, Rotterdam, The Netherlands
}

Objective To investigate changes in the incidence and treatment of prostate cancer over the period in which new diagnostic tools were introduced and the attitude towards treatment was changing.

Patients and methods Information on the extent of disease and treatment of patients diagnosed with prostate cancer within the Rotterdam region was retrieved from the Rotterdam Cancer Registry.

Results In the period 1989-95,4344 patients were diagnosed with prostate cancer and the age-standardized incidence increased from 62 to 125 per 100000 men. This increase mainly comprised tumours localized to the prostate, while the incidence of advanced cancers remained stable. The proportion of poorly differentiated tumours decreased from 33\% in 1989 to $24 \%$ in 1995 . In the same period the number of patients receiving radiotherapy increased from 80 to 258 , while the annual number of radical prostatectomies rose from 17 to 159 . Radiotherapy was the preferred type of treatment in patients over 70 years of age, whereas radical prostatectomy was used more frequently in younger patients with localized tumours.

Conclusion While the value of screening for prostate cancer remains in debate, incidence and treatment patterns are changing rapidly. Information on patterns of care is needed to interpret future mortality data and to plan resources for adequate health care.

Keywords prostate cancer, incidence, radical prostatectomy, radiotherapy, PSA

\section{Introduction}

In many western countries prostate cancer is rapidly becoming the most frequent cancer in men, as it already is in the USA [1,2]. Within Europe, the highest incidence rates are reported from Sweden, where prostate cancer has become more common than lung cancer [3]. Other European countries showing high incidence rates are Austria, Finland and the Netherlands. The age-standardized incidence of prostate cancer in the USA is about two to three times higher than in Europe [2, 4]. During the 1970 s and 1980s the age-standardized incidence in Europe increased by $5-25 \%$ every 5 years [ 5 ]. In the USA the increase was $15-25 \%$ every 5 years, until there was an 'epidemic' increase in the last decade; increases from $40-300 \%$ have recently been reported [6-10].

This 'epidemic' of prostate cancer has been induced by the introduction of new diagnostic methods [9, 10]. Because there are no curative treatment options for patients with advanced cancer, scientific attention has focused on the diagnosis and control of prostate cancer at an early stage. New tools such as TRUS and the biopsy gun facilitated the diagnosis of prostate cancer by needle

Accepted for publication 2 November 1999 biopsy. In the last decade the introduction of the PSA assay has markedly increased the opportunities to detect early prostate cancer. Several randomized studies are currently assessing the value of the PSA assay as a screening instrument. In the absence of conclusive results from these studies, the value of screening for prostate cancer remains controversial.

It is conceivable that the harm caused by overdiagnosis and subsequent over-treatment may overshadow the benefits of early detection [11,12]. Advocates of early detection have indicated that the survival after aggressive treatment of localized carcinomas is good $[13,14]$, albeit that several observational studies show reasonable results with a watchful-waiting policy $[12,15,16]$.

Consequently, there is a worldwide variation in the management of localized prostate cancer. In the USA, radical prostatectomy is becoming as popular as radiotherapy [6]. In some countries, radiotherapy is more popular [17] whereas in other countries watchful waiting is preferred [18], or the diagnosis of asymptomatic cancers considered not worthwhile [19]. To investigate the treatment policy for localized prostate cancer in the Rotterdam region in a period of increasing incidence, we analysed population-based data from the 
Rotterdam Cancer Registry. The Rotterdam region is of particular interest because the city is one of the participants in the European Randomised Screening study for Prostate Cancer (ERSPC) [20].

\section{Patients and methods}

Information about incidence and treatment of patients with prostate cancer was obtained from the Rotterdam Cancer Registry; the registry was started in 1982 and covers the south-western part of The Netherlands. Since 1989, registration has been complete in the central part of the registration area, thus allowing the calculation of population-based incidence rates. This central part of the Rotterdam region comprises $\approx 1.5$ million inhabitants; of the 750000 men, $\approx 100000$ are aged 55-69 years, 25000 are aged $70-74$ and 30000 are older than 74 years.

In 1992 the Rotterdam section of the ERSPC commenced [21]. Men living in Rotterdam and aged 55-75 years were invited to participate in a study in which they would be randomized between a screening arm and a control group. The men in the screening arm provided blood for PSA assay, with a 4-year screening interval. At the end of 1995 the screening arm of the study comprised $\approx 4000$ men $(\approx 3 \%$ of the respective population in that age group). The cancer registry receives information on whether men have been diagnosed after regular care or after screening.

All patients with prostate cancer diagnosed from 1989 to 1996 were included $(n=4344)$, except those with tumours incidentally found at autopsy $(n=33)$. Newly diagnosed patients with cancer are notified through notes from pathology laboratories and hospital discharge diagnoses. Trained registration clerks collect data from the clinical case-notes. Prostate cancers diagnosed from 1989 to 1993 were classified according to the 1987 rules of the UICC. Tumours diagnosed after 1992 were classified according to the UICC classification of 1992. The main change was the introduction of the T1c group for tumours identified as a result of PSA testing. For the present study, the clinical TNM information was combined, to group the tumours into four stages (Table 1). Clinical stages 1-3 can be considered to be potentially curable and are termed 'localized carcinomas', whereas stage 4 is termed 'advanced'. Tumours for which information on the clinical T-status was missing were referred to as stage $X$. The grade of differentiation of the tumours was coded as well-differentiated (grade 1 or Gleason score 2-4), moderately differentiated (grade 2 or Gleason score 5-7), poorly or undifferentiated (grade 3/4 or Gleason score 8-10) [22]. Tumour grade was based on the histological examination of the prostatectomy or biopsy specimen. Information on initial treatment was gathered for all patients. Only radical prostatectomies were evaluated as the surgical treatment. Transurethral resection and open prostatectomy for obstructive complaints with no resection of the lymph nodes were disregarded. Radiotherapy was only indicated when directed at the primary tumour with curative intent. Patients receiving postoperative radiotherapy $(n=23)$ were included in the surgery group. Since 1993 the PSA level at diagnosis has been recorded. Within the region, several kits for PSA determination have been used, but the Hybritech assay (Tandem, Hybritech Europe SA, Liege, Belgium) was the most common. Cases with an unknown PSA value $(n=275)$ were excluded from the analysis of PSA distribution.

Incidence rates were calculated by mode of detection (diagnosed through regular care or in the screening arm of the ERSPC). Age-standardization was conducted according to the European standard. Distributions of tumour stage and grade of differentiation were examined by year of diagnosis. Differences in grade distribution were analysed with the Pearson chi-square statistic. The distribution of PSA values was analysed for the total group and by tumour stage. The distribution of log PSA was evaluated statistically using an ANova. The evaluation of curative treatment for localized prostate cancer comprised overall trends and trends in different age groups. Surgery and radiotherapy were evaluated by age group, period of diagnosis and tumour stage.

\section{Results}

The age standardized incidence rate of prostate cancer increased from 62 to 125 per 100000 men, surpassing lung cancer as the most frequent form of cancer in the region. Only a small proportion of these cancers had been detected by the ERSPC study (Fig. 1). The proportion of cases detected by screening was 16\% in 1995 .

The increase was mainly in tumours that were confined to the prostate, especially those classified as stage 2 (Fig. 2). The annual number of stage 2 lesions increased from 134 in 1989 to 573 in 1995, comprising $29 \%$ and $58 \%$ of all tumours. Surprisingly, there was only a limited increase of stage 1 tumours, although the proportion of T1c tumours increased from 4\% in 1993 to

Table 1 Stage grouping according to the clinical TNM stage

\begin{tabular}{llll}
\hline Stage group & $c T$ & $c N$ & $c M$ \\
\hline 1 & $0,1,1 \mathrm{a}, 1 \mathrm{~b}, 1 \mathrm{c}$ & $0, \mathrm{X}$ & $0, \mathrm{X}$ \\
2 & $2,2 \mathrm{a}, 2 \mathrm{~b}, 2 \mathrm{c}$ & $0, \mathrm{X}$ & $0, \mathrm{X}$ \\
3 & $3,3 \mathrm{a}, 3 \mathrm{~b}, 3 \mathrm{c}$ & $0, \mathrm{X}$ & $0, \mathrm{X}$ \\
4 & $4,4 \mathrm{a}, 4 \mathrm{~b}$ & $1,2,3$ & $1,1 \mathrm{a}, 1 \mathrm{~b}, 1 \mathrm{c}$ \\
$\mathrm{X}$ & $\mathrm{X}$ & $0, \mathrm{X}$ & $0, \mathrm{X}$ \\
\hline
\end{tabular}


9\% in 1995 (data not shown). The number of advanced cancers remained stable throughout the study period, but declined proportionally from $30 \%$ to $16 \%$.

Despite the marked change in stage, the change in distribution of tumour grade was less apparent. In 1995 the proportion of poorly differentiated cancers was $23.7 \%$, compared with $33.3 \%$ in 1989 , whereas the proportion of well-differentiated cancers increased from $20.0 \%$ to $33.6 \%(P<0.001$; Table 2$)$. The median PSA value decreased from $27.0 \mathrm{ng} / \mathrm{mL}$ in 1993 to $15.1 \mathrm{ng} / \mathrm{mL}$ in $1995(P<0.001)$. There were no significant changes in PSA distribution within separate stage groups, except for a small decrease in the median PSA level for the group of stage 2 tumours, from 15.2 in 1993 to 13.0 in 1995 $(P<0.001$; Table 3).

The frequency of patients choosing definitive treatment changed considerably. From 1989 to 1995 the annual number of patients receiving radiotherapy increased from 80 to 258 , whereas the annual number of radical prostatectomies rose from 17 to 159 (Fig. 3); $95 \%$ of the patients receiving curative treatment had localized disease. Within the latter group, the proportion receiving curative treatment increased from $25.7 \%$ in 1989 (4.5\% surgery and $21.2 \%$ radiotherapy) to $52.1 \%$ in 1995 (20.1\% surgery and $32.0 \%$ radiotherapy).

The largest increase of the proportion of patients receiving curative treatment was in patients $<70$ years old (Fig. 4). For patients aged 70-74 years, there was also

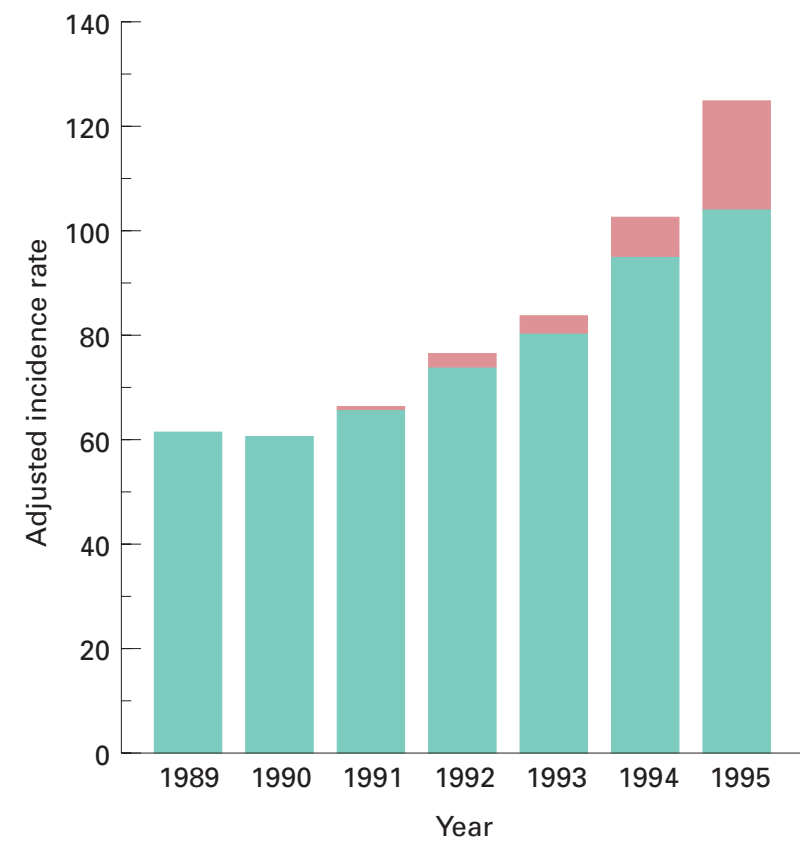

Fig. 1. The trend in incidence rates (per 100000 men, age-adjusted to the European population) according to the mode of detection (ERSPC, light red; regularly detected by the general health care system, light green). a considerable increase, but in patients over 74 years of age the proportion receiving curative treatment remained stable at about $20 \%$. Radiotherapy was the preferred treatment in patients $>70$ years of age, independent of stage (Table 4). For younger patients, radical prostatectomy has become the most common type of definitive treatment for stage 2 tumours. Tumour differentiation had a small influence on the frequency of curative treatment in patients with localized cancer. Treatment rates for patients with well, moderately and poorly differentiated tumours were $37 \%, 51 \%$ and $46 \%$, respectively.

\section{Discussion}

These results show that the treatment policy for localized prostate cancer has changed considerably. As in recent reports from the USA, the proportion of patients receiving definitive treatment has increased and radical prostatectomy has become the preferred treatment for patients $<70$ years old. Despite the growing role of surgery [23], radiotherapy has maintained a key role in the management of localized prostate cancer $[6,24]$.

Compared with patterns of care in the USA, several differences emerge. First, the age threshold for prostatectomy in the Rotterdam region is $\approx 70$ years, as against 75 years in the USA. Apparently, risks and benefits are considered to be different for men aged 70-75 and radiotherapy is still preferred by the regional urologists. Second, prostatectomy is mainly performed in patients with stage 2 disease, whereas studies in the USA report

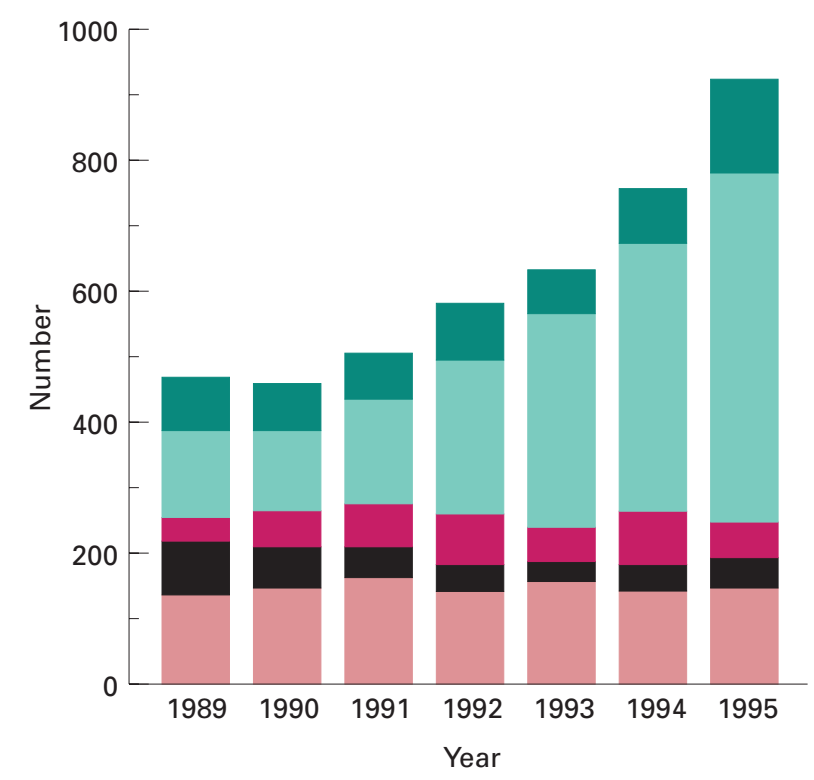

Fig. 2. The trend in the number of newly diagnosed carcinomas according to stage group (1 green; 2 light green; 3 red; 4 light red; $X$ black). 
Table 2 The tumour grade of differentiation, n (\%), according to year of diagnosis

\begin{tabular}{lllll}
\hline Year & Grade 1 & Grade 2 & Grade 3/4 & Grade unknown \\
\hline 1989 & $94(20.0)$ & $171(36.3)$ & $157(33.3)$ & $49(10.4)$ \\
1990 & $88(19.2)$ & $153(33.3)$ & $178(38.8)$ & $40(8.7)$ \\
1991 & $117(23.1)$ & $172(33.9)$ & $173(34.1)$ & $45(8.9)$ \\
1992 & $163(28.0)$ & $214(36.8)$ & $155(26.6)$ & $50(8.6)$ \\
1993 & $177(27.8)$ & $217(34.1)$ & $220(28.9)$ & $46(7.2)$ \\
1994 & $219(28.7)$ & $270(35.4)$ & $219(23.7)$ & $53(7.0)$ \\
1995 & $311(33.6)$ & $332(35.9)$ & & $64(6.9)$ \\
\hline
\end{tabular}

higher proportions of stage 3 cancers [25]. However, results related to stage are difficult to compare because the Rotterdam data refer to clinical stage, whereas USA registries combine information on clinical and pathological stage of disease. Moreover, clinical staging is subjective and additional staging procedures tend to vary both nationally and internationally. For clinical stage 3, radiotherapy is apparently preferred over surgery, although some tumours may prove to be confined to the prostate at operation. Whether radiotherapy for stage 3 should be considered as 'given with curative intent' is open to debate, but the 5-year survival for stage 3 cancers is $70 \%$ in the Netherlands [26]. A third difference from the situation in the USA is that in the Rotterdam region more patients are treated expectantly, especially in the oldest group. The efficacy of aggressive treatment for localized prostate cancer is still debated as the 10-year survival may be similar with initially conservative management $[15,16]$. Within the Rotterdam region, guidelines advocate expectant management for men who have a life expectancy of $<10$ years or an incidental finding of a well-differentiated tumour.

Several developments have contributed to the major rise in prostatectomy rates. The introduction of the PSA assay, supported by the introduction of TRUS and TRUSguided transrectal needle biopsies, has enabled the diagnosis of prostate cancer to be made at a stage where it is still curable. Although it is advisable to refrain from screening until the results from controlled randomized trials, e.g. the ERSPC in Europe and the PCLO in the USA, have been analysed and published, the PSA assay has evidently become routine in clinical practice. This has led to a dramatic increase in the incidence of prostate cancer. Even after excluding ERSPC-detected cases, the incidence still increased by $70 \%$ during the 7 -year study

Table 3 The distribution of PSA values $(\mathrm{ng} / \mathrm{mL}$ ) by year of diagnosis and stage

\begin{tabular}{|c|c|c|c|c|c|}
\hline \multirow[b]{2}{*}{ Stage/year } & \multicolumn{5}{|c|}{ Percentile } \\
\hline & 10 & 25 & 50 & 75 & 90 \\
\hline \multicolumn{6}{|l|}{ Total } \\
\hline 1993 & 4.6 & 10.2 & 27.0 & 83.0 & 315.2 \\
\hline 1994 & 4.1 & 8.6 & 21.1 & 58.0 & 182.4 \\
\hline 1995 & 4.0 & 7.1 & 15.1 & 45.0 & 179.6 \\
\hline \multicolumn{6}{|l|}{1} \\
\hline 1993 & 1.6 & 4.8 & 9.4 & 37.0 & 67.0 \\
\hline 1994 & 1.8 & 3.4 & 7.7 & 14.0 & 31.1 \\
\hline 1995 & 2.8 & 5.1 & 8.0 & 16.2 & 35.4 \\
\hline \multicolumn{6}{|l|}{2} \\
\hline 1993 & 4.5 & 8.7 & 15.2 & 41.8 & 89.5 \\
\hline 1994 & 4.2 & 8.5 & 18.0 & 40.3 & 89.0 \\
\hline 1995 & 3.6 & 6.7 & 13.0 & 29.0 & 54.0 \\
\hline \multicolumn{6}{|l|}{3} \\
\hline 1993 & 4.8 & 14.7 & 29.5 & 69.1 & 195.8 \\
\hline 1994 & 5.4 & 10.4 & 23.6 & 55.8 & 134.8 \\
\hline 1995 & 4.4 & 8.0 & 24.5 & 62.0 & 140.4 \\
\hline \multicolumn{6}{|l|}{4} \\
\hline 1993 & 20.8 & 45.5 & 108.5 & 378.8 & 1000 \\
\hline 1994 & 9.6 & 33.8 & 92.8 & 340.5 & 1000 \\
\hline 1995 & 13.0 & 51.0 & 160.0 & 498.0 & 1320 \\
\hline
\end{tabular}




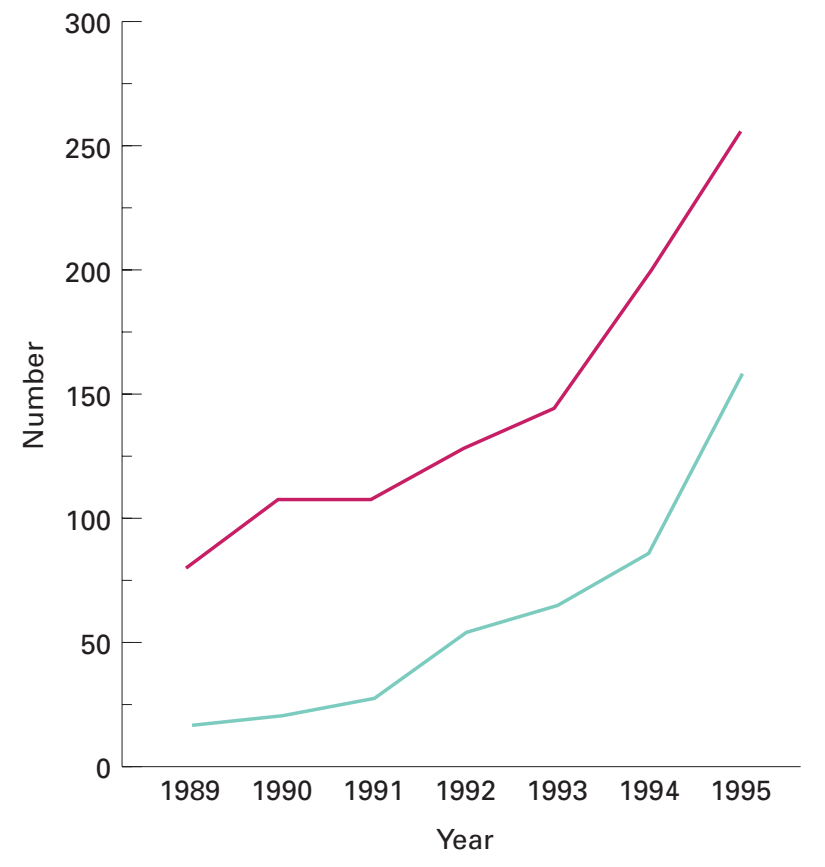

Fig. 3. The trend in the frequency of radical prostatectomy (green) and radiation treatment (red).

period. Another trend that should not be overlooked is the growing popularity of radical prostatectomy among urologists. Since the nerve-sparing procedure has improved, the outcome is considered more acceptable for potency and continence. In addition, more urologists have learned to carry out radical prostatectomies during residency and they are obviously more inclined to recommend therapeutic interventions which reflect their particular discipline. A widespread belief has grown that the results of radical prostatectomy in terms of long-term survival are superior to the other treatment modalities such as radiation and watchful waiting [27].

The clinical significance of screening-detected tumours, and therefore the desirability of aggressive treatment of these tumours, is much debated. Prostate cancer may be viewed as a pyramidal 'iceberg of disease' [28]. The tip of clinical or symptomatic disease projects above the surface, but there is a huge base of asymptomatic lesions which, under ordinary circumstances, lie hidden. Because this cancer base expands below the surface, screening tests can detect many cancers. With current knowledge it is not possible to predict whether these early cancers will become symptomatic or might have remained hidden as latent tumours.

In spite of the above, the present results suggest that clinically relevant tumours are being diagnosed. The increase in detection was not confined to T1c tumours, as might have been expected, but mainly comprised stage 2 cancers, i.e. these tumours were already palpable and/or

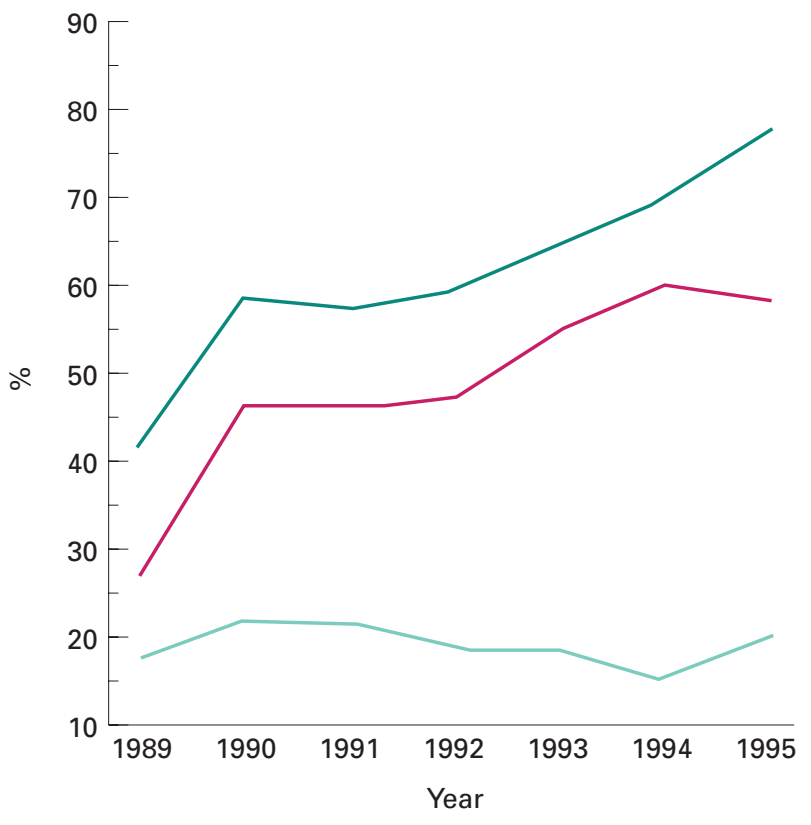

Fig. 4. The trend in the proportion of patients with localized prostate carcinomas who received curative therapy, by age group $(<74$ years, green; 70-74, red; $>74$, light green).

visible on TRUS or CT. Moreover, if these localized tumours were to be less aggressive, the stage shift would be reflected by a proportional decrease in poorly differentiated tumours. This idea is not supported by the minor shift from poorly differentiated to welldifferentiated tumours, although it was statistically significant because the sample was large. Also, within the various stage groups, the median PSA values only showed a minimal decline, suggesting that these localized tumours would probably behave aggressively.

In conclusion, the incidence and treatment patterns of prostate cancer are changing rapidly, and this will hopefully lead to a decrease in mortality. Future trends in mortality will be hard to explain without information on incidence patterns and treatment policy. Unfortunately, information on patterns of care within European countries is largely lacking. Although surveys among urologists may also be informative [28], populationbased data, like those presented here, and the pattern-ofcare studies in the USA, are urgently needed to interpret trends in mortality, and to plan and organize adequate healthcare.

\section{Acknowledgements}

We thank the members of the Regional Urological Tumour Working Party for their cooperation and especially Dr J.H.M. Blom for his valuable comments. 
Table 4 The percentage of patients with localized disease treated with curative intent (surgery or radiotherapy) according to clinical stage, age and period of diagnosis

\begin{tabular}{|c|c|c|c|c|c|c|}
\hline \multirow[b]{3}{*}{ Stage/treatment } & \multicolumn{6}{|l|}{ Age (years) } \\
\hline & \multicolumn{2}{|l|}{$<70$} & \multicolumn{2}{|l|}{$70-74$} & \multicolumn{2}{|l|}{$>74$} \\
\hline & 1989-1992 & 1993-1995 & 1989-1992 & 1993-1995 & 1989-1992 & 1993--1995 \\
\hline $1(n)$ & 78 & 119 & 98 & 67 & 135 & 108 \\
\hline Surgery & 2.6 & 23.5 & 0.0 & 1.5 & 0.0 & 0.9 \\
\hline Radiotherapy & 17.9 & 27.7 & 18.4 & 14.9 & 10.4 & 10.2 \\
\hline $2(n)$ & 216 & 444 & 139 & 324 & 308 & 509 \\
\hline Surgery & 30.6 & 44.4 & 10.8 & 14.2 & 1.3 & 1.8 \\
\hline Radiotherapy & 35.2 & 34.7 & 45.3 & 54.0 & 23.4 & 17.5 \\
\hline $3(n)$ & 73 & 71 & 53 & 47 & 102 & 73 \\
\hline Surgery & 13.7 & 18.3 & 5.7 & 2.1 & 0.0 & 0.0 \\
\hline Radiotherapy & 56.2 & 63.4 & 62.3 & 66.0 & 30.4 & 31.5 \\
\hline
\end{tabular}

\section{References}

1 Boyle P, Maisonneuve P, Napalkov P. Geographical and temporal patterns of incidence and mortality from prostate cancer. Urology 1995; 46: 47-55

2 Merrill RM, Potosky AL, Feuer EJ. Changing trends in U.S. prostate cancer incidence rates. J Natl Cancer Inst 1996; 88: 1683-5

3 Black RJ, Bray F, Ferlay J, Parkin DM. Cancer incidence and mortality in the European Union: cancer registry data and estimates of national incidence for 1990. Eur J Cancer 1997; 33: $1075-107$

4 Mettlin C. Clinical oncology update: prostate cancer, recent developments in the epidemiology of prostate cancer. Eur J Cancer 1997; 33: 340-7

5 Coleman MP, Estève J, Damiecki P, Arslan A, Renard H. Trends in cancer incidence and mortality. IARC Scientific Publications no. 1211993

6 Lu-Yao GL, Greenberg ER. Changes in prostate cancer incidence and treatment in USA. Lancet 1994; 343: 251-4

7 Gilliland FD, Welsch DJ, Hoffman RM, Key DJ. Rapid rise and subsequent decline in prostate cancer incidence rates for New Mexico 1989-93. Cancer Epidemiol Biomarkers Prev 1995; 4: 797-800

8 Stephenson RA, Smart CR, Mineau GP, James BC, Janerich DT, Dibble LR. The fall in incidence of prostate carcinoma: on the down side of a prostate antigen induced peak in incidence, data from the Utah Cancer Registry. Cancer 1996; 77: 1342-8

9 Potosky AL, Miller BA, Albertsen PC, Kramer BS. The role of increasing detection in the rising incidence of prostate cancer. JAMA 1995; 273: 548-52

10 Jacobsen SJ, Katusic SK, Bergstralh EJ et al. Incidence of prostate cancer diagnosis in the eras before and after serum prostate-specific antigen testing. JAMA 1995; 274: 1445-9

11 Abbas F, Scardino PT. The natural history of clinical prostate carcinoma. Cancer 1997; 80: 827-33

12 Fleming C, Wasson JH, Albertsen PC, Barry MJ, Wennberg JE. A decision analysis of alternative treatment strategies for clinically localized prostate cancer. JAMA 1993; 269: 2650-8

13 Smitt MC, Heltzel M. The results of radical prostatectomy at a community hospital during the prostate specific antigen era. Cancer 1996; 77: 928-33

14 Gerber GS, Thisted RA, Scardino PT et al. Results of radical prostatectomy in men with clinically localized prostate cancer. JAMA 1996; 276: 615-9

15 Lu-Yao GL, Yao SL. Population-based study of long-term survival in patients with clinically localised prostate cancer. Lancet 1997; 349: 906-10

16 Chodak GW, Thisted RA, Gerber GS et al. Results of conservative management of clinically localized prostate cancer. N Engl J Med 1994; 330: 242-8

17 McCredie M, Bell J, Lee A, Rogers J. Differences in patterns of care of prostate cancer, New South Wales, 1991. Aust NZ J Surg 1996; 66: 727-30

18 Chamberlain J, Melia J, Moss S, Brown J. Report prepared for the Health Technology Assessment panel of the NHS Executive on the diagnosis, management, treatment and costs of prostate cancer in England and Wales. Br J Urol 1997; 79: 1-32

19 Tretli S, Engeland A, Haldorsen T et al. Prostate cancer look to Denmark? J Natl Cancer Inst 1996; 88: 128

20 Schröder FH, Bangma CH. The European randomized study of screening for prostate cancer (ERSPC). Br J Urol 1997; 79: $68-71$

21 Schröder FH, Damhuis RAM, Kirkels WJ et al. European randomized study of screening for prostate cancer: the Rotterdam pilot studies. Int J Cancer 1996; 65: 145-51

22 Gleason DF, Mellinger GT. Prediction of prognosis for prostatic adenocarcinoma by combined histological grading and clinical staging. J Urol 1974; 11: 58-64

23 Lu-Yao GL, McLerran D, Wasson J, Wennberg JE. An assessment of radical prostatectomy: time trends, geographic variation, and outcomes. JAMA 1993; 269: 2633-6

24 Mettlin CJ, Murphy GP, McGinnis LS, Menck HR. The national cancer data base report on prostate cancer. Cancer 1995; 76: 1104-12 
25 Mettlin CJ, Murphy GP, Cunningham MP, Menck HR. The national cancer data base on race, age, and region variations in prostate cancer treatment. Cancer 1997; 80: 1263-6

26 Visser O, Schouten LJ, Dijck van JAAM, Coebergh JWW, eds. Urological Tumours in the Netherlands, 1989-96. Utrecht: Association of Comprehensive Cancer Centres, 1999

27 Whitmore WF Jr. Management of clinically localized prostate cancer, an unresolved problem. JAMA 1993; 269: $2676-7$

28 Kramer BS, Gohagan JK, Prorok PC. Is screening for prostate cancer the current gold standard? No! Eur J Can 1997; 33: 348-53
29 Savage P, Bates C, Abe P, Waxman J. British urological surgery practice. 1. Prostate Cancer. Br J Urol 1997; 79: 749-54

\section{Authors}

S.J.J.C. Spapen, Epidemiologist.

R.A.M. Damhuis, MD, Epidemiologist.

W.J. Kirkels, Urologist.

Correspondence: R.A.M. Damhuis, Comprehensive Cancer Centre Rotterdam, PO Box 289, 3000 AG Rotterdam, The Netherlands. E-mail: canreg@ikr.nl 